EESTI NSV TEADUSTE AKADEEMIA TOIMETISED. 25. KOIDE FOOSIKA * MATEMAATIKA. 1976, NR. 3

ИЗВЕСТИЯ АҚАДЕМИИ НАУК ЭСТОНСКОИ ССР. ТОМ 25 ФИЗИКА * МАТЕМАТИКА. 1976, № 3

Ю. ЛИИВАК

\title{
К ОПРЕДЕЛЕНИЮ ФУНКЦИОНАЛЬНОЙ ЭФФЕКТИВНОСТИ СЕТЕЙ ЭВМ
}

J. LIIVAK. ARVUTITEVAHELISTE VORKUDE FUNKTSIONAALSE EFEKTIIVSUSE MAARAMISEST

J. LIIVAK. A NOTE ON THE FUNCTIONAL EFFICIENCY COMPUTER OF NETWORKS

В данном сообщении анализируются факторы, влияющие на функциональную эффективность сети, и выводится критерий для оценки топологической оптимальности сети ЭВМ.

Сеть ЭВМ есть совокупность вычислительных центров и абонентских пунктов, соединенных с помощью подсети передачи данных, для предоставления потребителям возможности передачи, обработки и хранения данных в режиме разделения ресурсов. Ресурсами сети являются специальное и общее программное обеспечение, аппаратно-вычислительные возможности, а также автоматизированные банки данных.

В ходе анализа тенденции роста рынка на вычислительную технику в США выяснилось, что обработка и передача данных в автономном режиме все более интенсивно заменяются режимом оперативной обработки на ЭВМ. Предполагается, что к 1980 г. $70 \%$ абонентских пунктов (около 2 млн.) будут непосредственно связаны с ЭВМ [ $\left.{ }^{1}\right]$. Такая же тенденция пользования вычислительной техникой наблюдается и у нас, особенно в связи с разработкой и постепенным внедрением Государственной сети вычислительных центров СССР. Проектирование территориально распределенных сетей требует от разработчиков строго продуманного подхода к оптимизации сети. Однако критерии, предложенные в $\left[{ }^{2}\right]$, не связаны ни с выполняемыми сетью ЭВМ функциями, ни с основной задачей - обеспечением качества обрабатываемых в сети данных. Поэтому выбор критерия функциональной эффективности сети является первоочередной задачей. Мы считаем целесообразным исходить из следующего: выполняемые сетью функции должны обеспечить заданное качество данных при минимальных затратах. Ниже рассмотрим, как функциональная эффективность связана с выполняемыми сетью задачами, перечень которых дает таблица.

Качество данных определяют следующие свойства: 1) достоверность, 2) актуальность, своевременность, 3) релевантность, 4) полнота, 5) удобство восприятия, 6) содержательность.

Как показал анализ, проведенный Е. Г. Ясиным $\left[{ }^{3}\right]$, из них первые два свойства являются независимыми и наиболее важными с точки зрения качества сети, в то время как остальные свойства зависят друг от друга и не поддаются количественной оценке. 


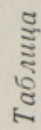

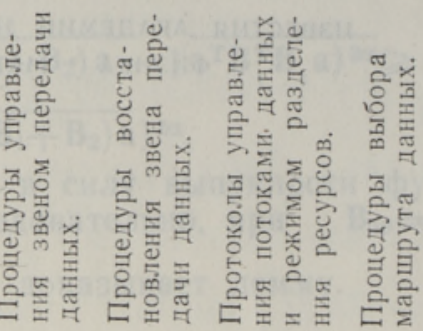

- $i$ ल

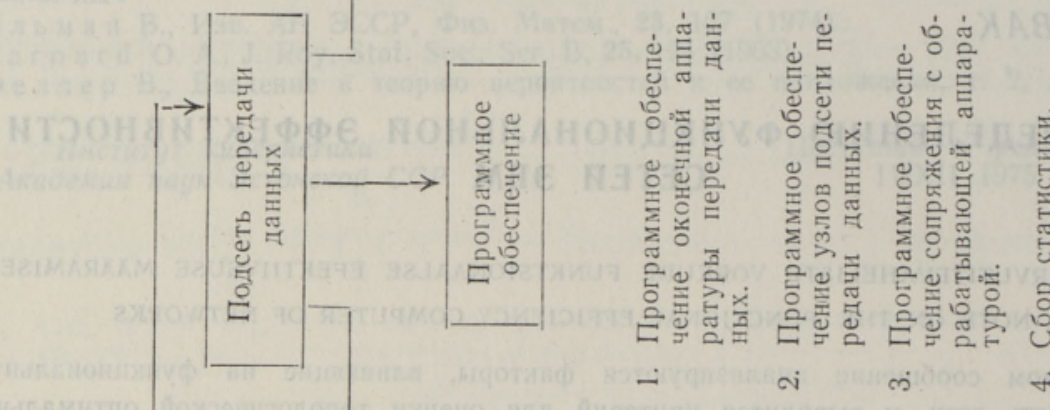

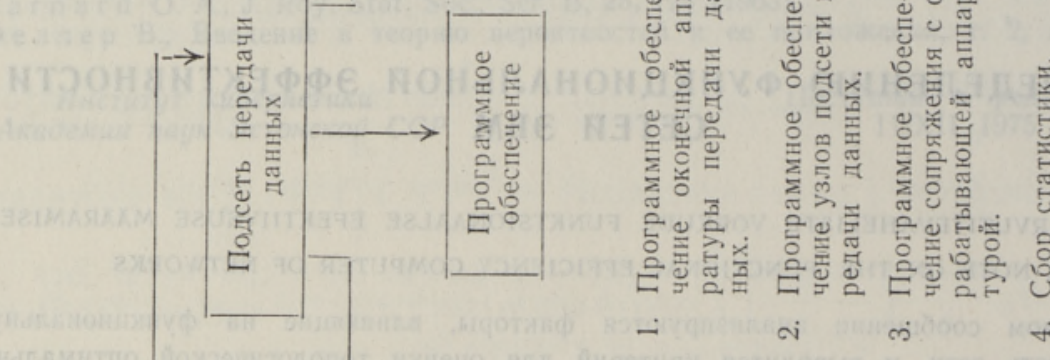

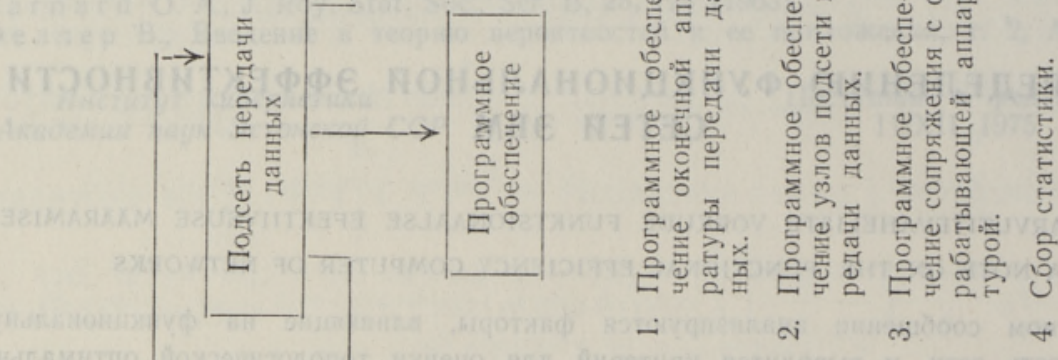

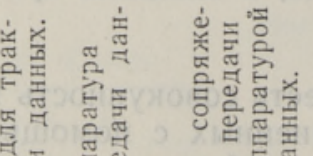

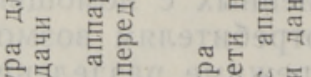

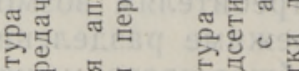

흥

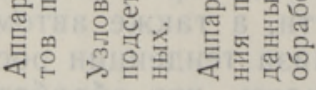

- i

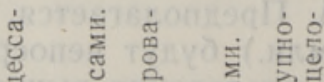

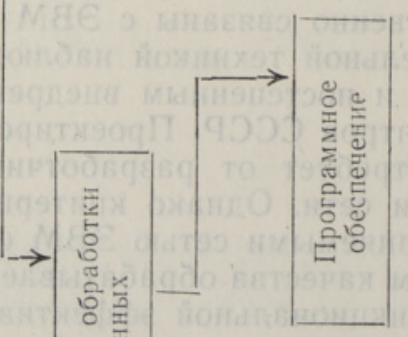

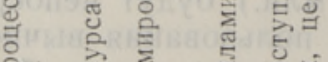

릴 离

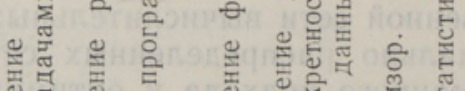

宓

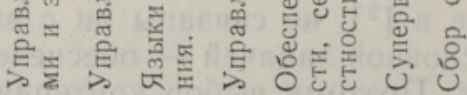

- ब म

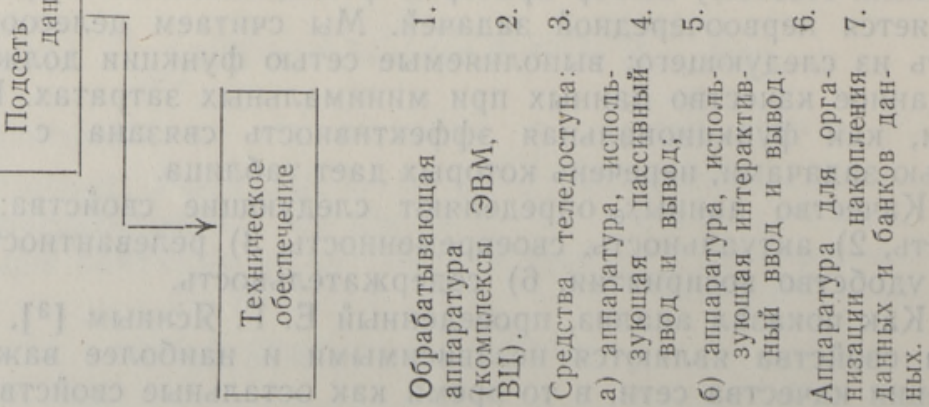


Достоверность данных определяется помехами в сети, к которым относятся следующие: 1) сбой и отказы, 2) статистический шум и помехи в канале связи, 3) семантический шум (ошибки интерпретации данных), 4) дезинформация (приписки, ошибки оператора и т. д.).

В зависимости от вида помех выбирают и соответствующие способы защиты. Семантический шум и дезинформация имеют, как правило, не случайный характер. Избежать их помогают организационные мероприятия, подходящая конструкция абонентских пунктов (например, возврат на источник данных ранее переданных в сеть символов); а в некоторых случаях и применение логико-программных средств. Остальные виды помех являются случайными и устранить их можно введением в структуру сети избыточности на всех уровнях (резервированием процессоров, введением аппаратного контроля, избыточным кодированием информации, применением мажоритарного принципа при построении схем и т. д.). Следовательно, достоверность данных обеспечивают следующие организационные и конструктивные меры: 1) помехоустойчивое кодирование и использование избыточности в алгоритмах обработки данных в сети и 2) эффективные и качественные методы контроля сети (как аппаратурные, так и логико-программные).

Актуальность и своевременность являются функциями от времени. Они зависят от времени ответа и времени передачи сообщения в сети ЭВМ, которые, в свою очередь, являются функциями от производительности комплексов ЭВМ, обрабатывающих данные, от быстродействия узловой аппаратуры и от эффективности логического обеспечения подсети передачи данных.

Таким образом, видим, что обеспечение качества данных является оптимизационной задачей: своевременность достигается за счет сокращения задержек в сети и повышения эффективной скорости передачи данных, а достоверность - за счет уменьшения эффективной скорости передачи данных.

Для определения функциональной эффективности рассмотрим сеть ЭВМ, ориентированную на коммутацию пакетов, что выгодно отличается от коммутаций каналов и сообщений с точки зрения обеспечения качества данных. Во-первых, пакеты из-за меньшего по сравнению с сообщениями размера менее подвержены воздействию случайных помех. Во-вторых, происходит сокращение задержек передачи данных по сети в результате экономии времени на обслуживание и, наконец, уменьшается объем буферной памяти и становится менее сложной аппаратура коммутаций.

Повышение качества данных в сети ЭВМ связано с затратами, включающими расходы на оборудование и эксплуатацию. Функциональную эффективность $E_{\Phi}$ можно описать следующей зависимостью:

$$
E_{\phi}=E\left(E_{\mathrm{o}}, E_{\mathrm{y}}, E_{\mathrm{k}}, E_{3}, E_{\mathrm{M}}, E_{\mathrm{I}}, C_{0}, C_{\ni}\right)\left[\frac{\text { пакетов }}{\text { сек }} \text { руб. }\right]
$$

где $E_{0}-$ скорость обработки пакетов данных на вычислительных центрах;

$E_{\text {y }}$ - скорость обработки пакетов данных, зависящая от быстродействия узловой аппаратуры;

$E_{\mathrm{k}}$ - количество пакетов в сети в единицу времени, зависящее от пропускной способности и длины каналов;

$E_{3}$ - эффективная скорость передачи пакетов данных с учетом процедур управления и восстановления звеном передачи данных; 
$E_{\mathrm{M}}$ - эффективная скорость передачи пакетов данных с учетом способа выбора маршрута;

$E_{\text {II }}$ - эффективная скорость передачи пакетов данных с учетом способов управления потоками и режимом разделения ресурсов;

$C_{0}$ - стоимость оборудования и каналов связи;

$C_{\text {э }}$ - эксплуатационные расходы.

Определение функциональной эффективности сети ЭВМ по приведенной выше зависимости является самостоятельной задачей для каждой конкретной сети.

Весьма важным моментом является и топологически рациональное построение сети ЭВМ, которое расширяет потенциальные возможности для эффективного и надежного ее функционирования. Топологически оптимальная сеть должна пропускать в единицу времени максимальное количество пакетов при минимальной стоимости каналов связи и гарантировать при этом качество данных. Критерий для оценки топологической оптимальности $\left(E_{\mathrm{T}}\right)$ представляет собой частный случай критерия функциональной эффективности. На основании (1) этот критерий можно представить в виде

$$
E_{\mathrm{T}}=E\left(E_{\mathrm{K}}, E_{\mathrm{M}}, C_{\mathrm{K}}\right)\left[\frac{\text { пакетов }}{\text { сек }} \text { руб. }\right] \text {, }
$$

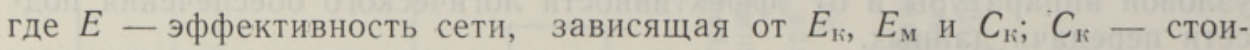
мость каналов связи.

\section{Л И ТЕР А Т У Р А}

1. Ch ou W., AFIPS Conf. Proc., 43, 1974, p. 553.

2. Железнев Н. А., В сб.: Использование избыточности в информационных си. стемах, Л., 1970 , с. 9.

3. Я с ин Е. Г., Экономика и матем. методы, 7, № 3, 389 (1971).

Институт кибернетики Академии наук Эстонской ССР
Поступила в редакцию 12/XII 1975 